\title{
A Comparison of Hypertrophy Potential between the Vastus Lateralis and Rectus Femoris during Level Walking in Combination with Vascular Restriction
}

JOSE A. LEAL Jr., CHARITY CAVAZOS, ROBERT CARDENAS, YOSHIAKI SATO, MICHAEL G. BEMBEN, AND MURAT KARABULUT

University of Texas at Brownsville/Texas Southmost College, Brownsville, TX, University of Oklahoma, Norman, OK.

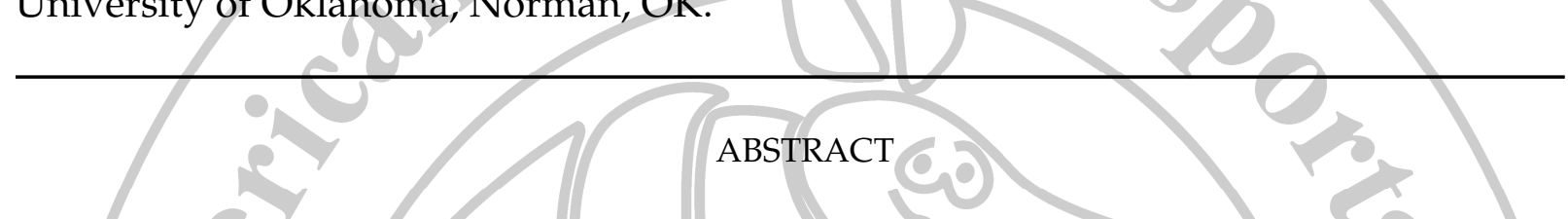

Recent research has found that by compressing blood flow to working muscle during lower body exercise involving short bouts of walk training results in an increased muscle volume and improved quadriceps strength. However, the effects of vascular restriction (VR) during walking on neuromuscular adaptations as assessed by electromyography (EMG) activity may provide insight on how low intensity training with vascular restriction can be applied to various modes of exercise. The purpose of this study was to explore and compare muscle activation changes of the quadriceps muscles during walking using EMG with and without blood flow restriction. EMG electrodes were placed along the longitudinal axis of the vastus lateralis (VL) and rectus femoris $(\mathrm{RF})$ of the right thigh of seven male volunteers (mean $\pm \mathrm{SD}$ age $=22.7 \pm$ 6.8 years; height $=178 \pm 5.4 \mathrm{~cm}$; weight $=82.6 \pm 7.7 \mathrm{~kg}$ ). Placement of the electrode was marked at a point of $33.3 \%$ on VL, and RF $50 \%$ of the distance between the lateral femoral epicondyle and the greater trochanter. On two separate testing sessions, subjects walked on a treadmill for two, 10 minutes bouts separated by a $5 \mathrm{~min}$ rest period at a speed of $80.4 \mathrm{~m}^{*} \mathrm{~min}^{-1}$ with and without blood flow restriction. There were significant main effects for both condition (with and without VR) and muscles (VL and RF), as well as an interaction between condition and muscles (condition*muscle) for EMG amplitude (RMS). On the other hand, there were no significant main effects or interactions for EMG median frequency (MDF). The findings suggest that walking with VR might cause neural adaptation in both VL and RF, but the VL might have a better potential for hypertrophic response when performing slow walking with blood flow restriction. Walking speed and the pressure used for compression during walking might change the pattern of response, therefore, both need to be given careful consideration in the design of future studies.

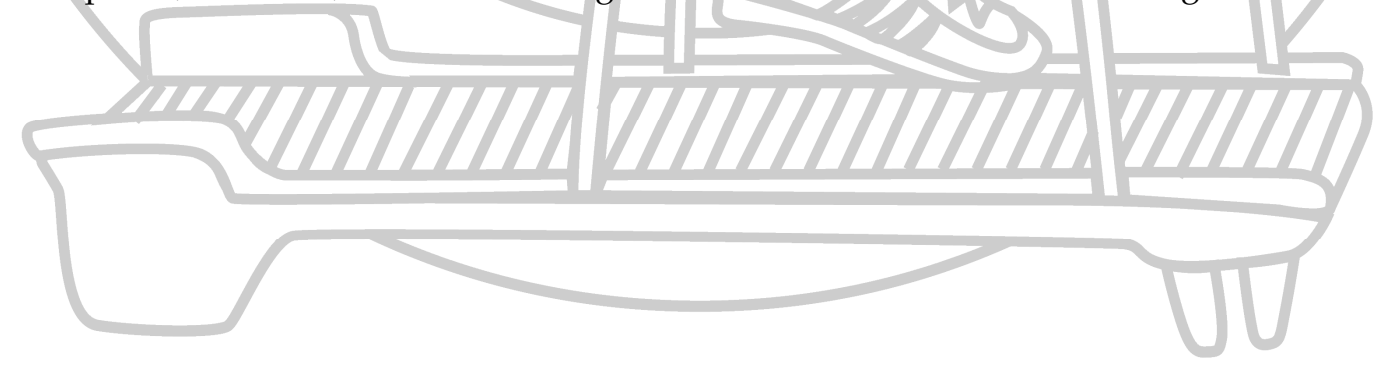

